Unfallchirurg 2014 · 117:974-974

DOI 10.1007/s00113-014-2646-8

Online publiziert: 15. Oktober 2014

(c) Springer-Verlag Berlin Heidelberg 2014

\title{
C. Krettek
}

Unfallchirurgische Klinik, Medizinische Hochschule Hannover

\section{Schussverletzungen}

Sehr geehrte Leserinnen und Leser,

Schussverletzungen sind erfreulicherweise selten in Deutschland, können aber gravierende Folgen haben. Schussverletzungen weisen hinsichtlich Genese, Gewebeschaden und Komplikationsträchtigkeit wichtige Unterschiede gegenüber den Verletzungsmustern nach stumpfen Verletzungen wie z. B. infolge von Hochrasanztraumen auf. Neben ausgedehnten Weichgewebe- und Knochendefekten, Kontamination und konsekutiver Keimbesiedelung besteht bei Schuss-, Explosions- und Verbrennungswunden das Problem der sogenannten "developing wounds“. Dies bedeutet, dass diese Wunden ihre eigene Kinetik und Dynamik haben und sich der Gewebeschaden auf einer Zeitachse innerhalb von mehreren Tagen weiterentwickeln und ausdehnen kann.

Einschätzung und Behandlung von Schussverletzungen an der unteren Extremität sind damit anspruchsvolle Aufgaben, die in die Hand eines erfahrenen Unfallchirurgen gehören. Die Qualität seiner Erstversorgung und seine Erfahrungen entscheiden wesentlich über Erhalt der Extremität und das erreichbare Endergebnis.

Ausgewiesene Chirurgen aus Bundeswehreinrichtungen in Koblenz und Hamburg stellen in sehr lesenswerten Leitthemenartikeln den allerneuesten Wissensstand zu Pathophysiologie und Behandlung von Schussverletzungen dar, in die die Erfahrungen aus den jüngsten Militäreinsätzen eingeflossen sind.

Verletzungen durch Schuss- und Stichwaffen in Deutschland sind überwiegend mit Gewaltverbrechen und Suizidversuchen assoziiert. Abhängig von der verletzten Körperregion weisen sie eine hohe Mortalität auf und sind oft mit kreis- laufrelevanten Blutungen vergesellschaftet. In einem weiteren Artikel werden aktuelle epidemiologische Daten zu Schussund Stichverletzungen in Deutschland präsentiert und analysiert.

Die Merkmalkombination "selten“ und "folgenschwer" bringt es mit sich, dass die Vorbereitung auf diese Verletzung notwendig, aber auch nicht zuletzt durch den Mangel an täglicher Routine schwierig ist. Die nachfolgenden 3 Artikel zu diesem Themenkomplex sollen den Leser sensibilisieren, informieren und vorbereiten auf Situationen, die zumindest bei uns erfreulicherweise weiter rückläufig sind.

Ihr

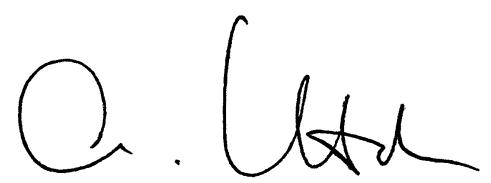

C. Krettek

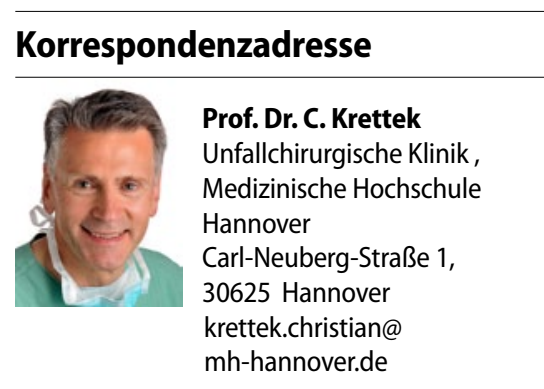

Einhaltung ethischer Richtlinien

Interessenkonflikt. C. Krettek gibt an, dass kein Interessenkonflikt besteht. 